\title{
François-Marie Raoult et la loi de Raoult
}

\section{3 mai 1887 : François-Marie Raoult découvre une propriété universelle des molécules dissoutes et montre comment l'utiliser.}

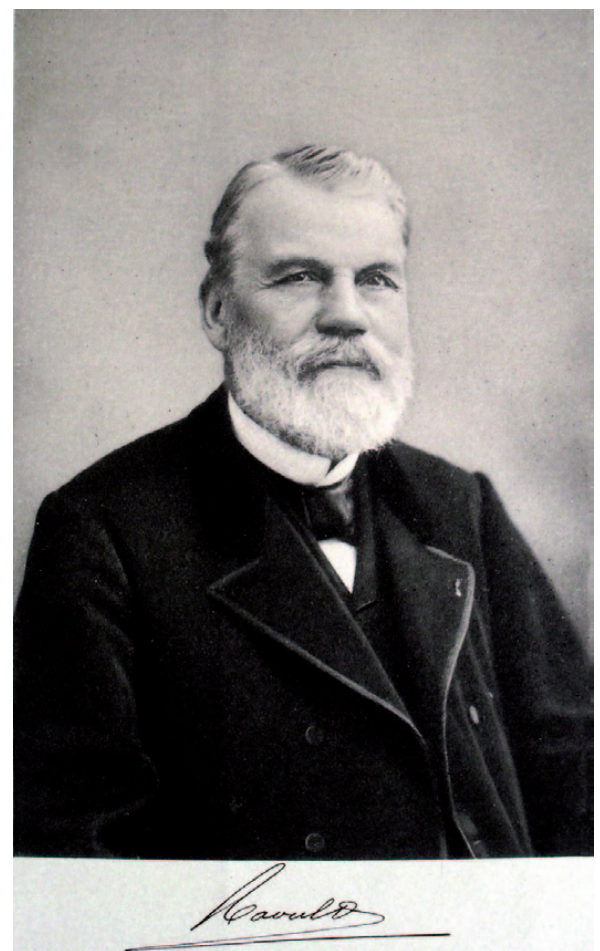

François-Marie Raoult (1830-1901)

(Source : Zeitschrift fuir physikalische chemie 27 (1898)).
Nous remercions Alan Chodos, éditeur d'APS-News, qui nous a autorisés à publier la traduction de l'article paru dans la rubrique "This Month in Physics History" du numéro 20 (mai 2011).

Traduction: C.H. de Novion et A. Cymer.

\section{Références}

• F.M. Raoult, « Lois générales de l'ébulliométrie », Comptes Rendus 104 (1887) 1430-3.

2• M.J. Nye, Science in the Provinces: Scientific Communities and Provincial Leadership in France, 1860-1930, University of California Press (1986).
Au début du $19^{\text {e }}$ siècle, la théorie atomique et moléculaire de la matière ne pouvait être complètement acceptée que dans la mesure où l'on était capable de relier les propriétés macroscopiques familières aux propriétés des molécules individuelles. Ceci a commencé à se faire quand Amedeo Avogadro proposa en 1811 que, dans des conditions identiques, tous les gaz contiennent le même nombre de molécules par unité de volume. Sur ces bases, la théorie des gaz parfaits a suscité pléthore d'activités et d'avancées en physique. Dans un gaz parfait, les molécules de toute nature se comportent de la même manière : le caractère universel de ce concept montre qu'il existe une physique sous-jacente qui décrit une grande partie du monde matériel. Par la suite, les physiciens trouvèrent le moyen de déterminer la constante d'Avogadro, c'est-à-dire le nombre exact de molécules dans un volume donné. Les masses moléculaires ont alors pu être déterminées simplement, en mesurant la densité d'un gaz.

Mais notre compréhension des gaz a fait peu avancer celle des liquides, des solutions et de la matière condensée. La plupart des molécules ne peuvent être facilement observées sous la forme de gaz. En revanche, dissoutes dans un liquide, leur comportement collectif peut être observé, comme dans la théorie des gaz parfaits. Pour cela, il a fallu attendre le travail d'un physico-chimiste français, François-Marie Raoult.

\section{La loi de Raoult}

Après des années d'expériences soigneuses, Raoult publie dans les Comptes rendus (23 mai 1887) un article décisif : "Loi générale de l'ébulliométrie » [1], où l'on retrouve l'idée centrale de ce qui deviendra la loi de Raoult. Lors de ses expériences, il dissout de faibles concentrations de composés non volatils : 5 sortes dans l'eau, et 14 sortes dans 11 solvants organiques, puis il mesure l'effet des composés dissouts sur la pression de vapeur du solvant. Il trouve le résultat remarquable que la présence d'une fraction molaire donnée de n'importe quelle substance dissoute dans tout liquide résulte en un même taux de réduction de la pression de vapeur. Confirmées ultérieurement par d'autres, ces expériences fournissent le genre d'universalité qu'Avogadro et les architectes de la théorie des gaz parfaits souhaitaient donner à la physique de leur époque. Dans d'autres expériences, Raoult a montré un effet similaire pour la réduction du point de fusion d'un liquide par des substances dissoutes (fig. 1). Des travaux théoriques ultérieurs de Jacobus van't Hoff et Svante Arrhenius ont confirmé son résultat, en ont fourni les bases thermodynamiques et ont montré comment comprendre ce qui, au départ, semblait des exceptions. 
Concentration $(\%)$

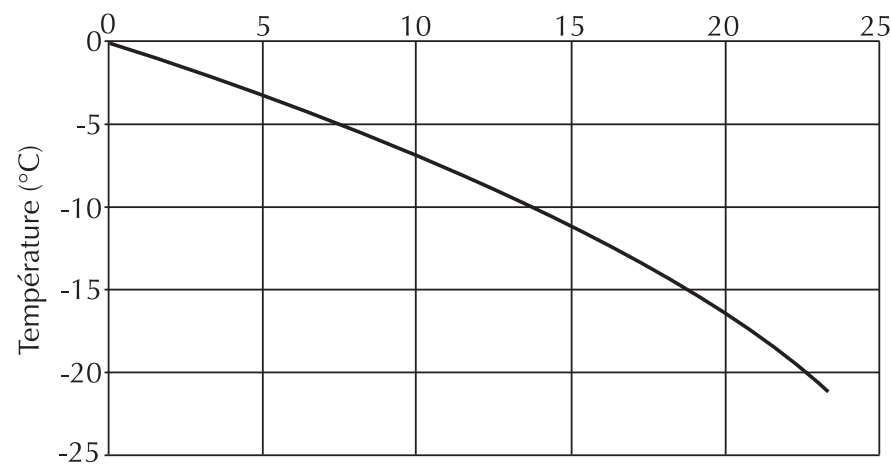

1. Illustration de la loi de la cryométrie de Raoult : abaissement de la température de congélation d'une solution aqueuse de chlorure de sodium, en fonction du pourcentage massique de sel dissout. La loi de la cryométrie de Raoult spécifie que l'abaissement de la température de congélation de la solution est proportionnel à la concentration molaire en soluté ; on remarquera la déviation expérimentale par rapport à cette loi à concentration élevée.

Le caractère universel de la loi de Raoult a suscité un engouement des scientifiques pour l'étude des liquides et des solutions. Pour une quantité donnée de matière dissoute de poids moléculaire inconnu (même élevé, comme dans le cas des polymères), la loi de Raoult permet de déterminer ce poids en mesurant la pression de vapeur du solvant.

La loi de Raoult et celle des gaz parfaits ne sont toutes deux valables que dans des domaines restreints de pression et de température. La loi des gaz parfaits implique des températures relativement élevées et des pressions basses ; à haute pression et basse température, elle ne s'applique plus : des gaz différents se comportent alors autrement, en suivant souvent l'équation de Van der Waals, et se condensant éventuellement en phase liquide. En explorant au-delà du domaine de validité de la loi originale, une physique très riche a été découverte.

La loi de Raoult, elle, s'applique dans la limite des basses concentrations et pour des solutés non volatils. Aux concentrations élevées, ou pour des solutés volatils, des composés différents se comportent de façon distincte. Explorer ces domaines a fourni de nombreuses données qui ont permis de concevoir la distillation fractionnée et d'autres procédés industriels. D'autre part, pour plusieurs sels, dont le chlorure de sodium dans l'eau, Raoult a trouvé un nombre de molécules dissoutes environ deux fois plus élevé que prévu, ce qui était un premier indice que le chlorure de sodium se dissout dans l'eau en ions sodium et chlore.

\section{Les premières années}

Raoult (1830-1901), fils d'un agent des douanes, est né à Fournes, dans le département du Nord. Il a étudié à l'Université de Paris pendant une courte période, mais manquait de ressources financières pour continuer. Il a ensuite passé 14 ans à enseigner la physique et les mathématiques dans plusieurs lycées, à plein temps et se déplaçant d'une école à l'autre. Simultanément, au prix de grandes privations, il a continué seul ses recherches sur la force électromotrice des piles électriques, qui lui ont permis d'obtenir le grade de docteur ès sciences physiques de l'Université de Paris. Bien que peu remarqué à l'époque, c'était un travail de pionnier. Raoult a été l'un des premiers à montrer que la chaleur de réaction chimique d'une pile voltaïque n'est pas identique à son énergie électrique. La reconnaissance de ce travail n'est venue que plus tard, lorsque les concepts thermodynamiques d'énergie libre et d'entropie furent compris et appliqués aux piles électrochimiques.

En 1867, Raoult postula à la faculté des sciences de Grenoble comme professeur de chimie. Il lui a été objecté qu'il était en fait un physicien, mais sa nomination fut finalement approuvée en 1870, et il put continuer son travail de recherche à Grenoble pendant 31 ans. À cette époque, le laboratoire était très rudimentaire. Dans son livre, Science in the Provinces [2], l'historienne Mary Jo Nye cite la description de Raoult : "Chaque professeur avait sa propre petite table. Cette pièce, qui était un laboratoire pendant le jour, servait en même temps de logement pour le concierge. Ici, le professeur de physique mettait en ordre ses instruments ; le professeur de zoologie disséquait ses lapins et nourrissait ses pigeons ; le professeur de géologie préparait ses fossiles ; et le professeur de chimie effectuait toutes ses manipulations. "

\section{L'héritage de Raoult}

La vie de François-Marie Raoult a été une longue bataille pour effectuer le travail qu'il aimait et pour voir ses mérites reconnus. Il a souffert de difficultés financières pour effectuer son doctorat, d'un long apprentissage de l'enseignement au lycée et de réticences avant d'être nommé professeur à l'Université de Grenoble. Mais, en fin de compte, il a obtenu une renommée méritée, d'abord à l'étranger, et finalement en France.

Richard Williams Auteur invité d'APS News 